\title{
Evaluación educativa en tiempo de pandemia de COVID-19. ¿Cómo cambió en los ámbitos hospitalarios? Una propuesta
}

\section{Educational evaluation in time of the COVID-19 pandemic. How do I change in hospital settings? A proposal}

\author{
Juan Carlos Vázquez-Minero, ${ }^{* \neq}$ María de Jesús Villalba-Calderón,* Arturo Chávez-Tinoco ${ }^{\ddagger}$
}

*Facultad de Medicina, Universidad Nacional Autónoma de México.

${ }^{\ddagger}$ Instituto Nacional de Enfermedades Respiratorias Ismael Cosío Villegas, Ciudad de México, México.

Palabras clave: Evaluación, evaluación hospitalaria, evaluación clínica, COVID-19.

Keywords: Evaluation, hospital evaluation, clinical evaluation, COVID-19.

\section{INTRODUCCIÓN}

En México, en el mes de febrero del año 2020, una de las epidemias más devastadoras de la historia moderna de la humanidad tocó a nuestra sociedad, el COVID-19 había llegado para quedarse por mucho tiempo. Comenzaron los cambios en la manera de trabajar y estudiar con la finalidad de protegernos del contagio, y así se abandonaron las escuelas, las aulas quedaron vacías, pero la vida académica no se podía detener, por lo que fue necesario reinventar la educación y aplicar la tecnología para ese fin. Se inició la época de la educación a distancia como la forma más importante de interacción entre los alumnos y los profesores, ya no había posibilidad del contacto humano, y esta educación que antes no era cotidiana, se convirtió en algo habitual para los diferentes niveles educativos. Esto también provocó que se tuvieran que cambiar todos los métodos y herramientas educativas y también las formas

Correspondencia:

Dr. Juan Carlos Vázquez-Minero

Instituto Nacional de Enfermedades Respiratorias Ismael Cosío

Villegas, Ciudad de México.

Correo electrónico: minerojc@hotmail.com

Recibido: 21-V-2021; aceptado: 25-V-2021.

Citar como: Vázquez-Minero JC, Villalba-Calderón MJ, Chávez-Tinoco A. Evaluación educativa en tiempo de pandemia de COVID-19. ¿Cómo cambió en los ámbitos hospitalarios? Una propuesta. Neumol Cir Torax. 2021; 80 (3): 204-207. https://dx.doi.org/10.35366/102481 de evaluar. Cada quien adoptó las que más conocían y eran más factibles de utilizar.

El ser humano desde que nace aprende de manera informal y no estructurada, adquiere sus primeros conocimientos en la escuela inicial de la familia, después ya de una forma estructurada en la escuela formal adquiere los conocimientos que le darán las bases para los futuros estudios. Pero es importante pensar cómo sabemos que estos conocimientos son adquiridos y aprovechados por los educandos. A este proceso lo conocemos como evaluación del aprendizaje. Como lo menciona Rowntree en 1977: evaluar es un intento de conocer a la persona. A lo largo de la historia de la educación un aspecto fundamental es la evaluación, es decir, la evaluación es inherente al acto educativo. Los métodos para llegar a conocer a la persona, esto es, para evaluarla son muy diversos y dependen del momento histórico de las sociedades y en este caso de las condiciones sociales que permitan determinada forma de evaluación.

La educación ha tenido que migrar a nuevos espacios educativos, la escuela cedió su espacio a ambientes como el hogar del estudiante, que fue transformado en aula y los medios digitales se convirtieron en un puente entre el docente y el alumno. A partir de la pandemia del COVID-19 que paralizó al mundo, las tecnologías se convirtieron en un conductor importante entre alumnos, padres, docentes e instituciones. Por tal motivo, se ha generado un cambio en el proceso de evaluación en el cual los docentes enfrentan un reto importante para generar nuevas alternativas al momento de evaluar.

En esta reflexión presentaremos algunas alternativas de evaluación aplicables a la educación de pregrado, que pueden ser útiles en los contextos hospitalarios limitados por el distanciamiento social y la sana distancia. 


\section{LA EVALUACIÓN EN LA EDUCACIÓN MÉDICA}

La evaluación como la propia educación ha tenido que ir cambiando con el paso de los años, su desarrollo se puede dividir en cuatro etapas o generaciones: en la primera generación, basada en los trabajos de Binet y Spearman, el objetivo fundamental era la medición de los resultados a través de pruebas. En la segunda generación de Tyler los resultados deberían servir para emitir juicios acerca del proceso de aprendizaje. En la tercera generación de Scriven la evaluación debe servir para mejorar la toma de decisiones en el proceso de aprendizaje. Y en la cuarta generación ya existe la investigación evaluativa. Después de todas estas bases en la evaluación de la educación, vinieron los cambios de enseñanza por la pandemia de COVD-19. Creemos que en esta pandemia se desarrolló de manera definitiva una quinta etapa, que es el de adaptar los recursos tecnológicos en la evaluación a distancia, teniendo esa parte de investigación y de procesos de mejora. ${ }^{1-3}$

La evaluación del aprendizaje de manera práctica se ha dividido en evaluaciones diagnósticas, las cuales se aplican al ingreso a un curso tratando de definir si el alumno tiene las competencias necesarias para cursarlo, la evaluación formativa, la cual se aplica en diferentes tiempos del proceso educativo y nos permite conocer el avance del alumno y hacer ajustes y correcciones de los cursos y por último, la evaluación sumativa que se aplica al final del proceso educativo con la finalidad de verificar los conocimientos, habilidades y actitudes del alumno al egresar., ${ }^{4,5}$

Pero todo esto tiene que partir del principio fundamental de ipara qué evaluamos? No se puede concebir el aprendizaje sin un proceso de evaluación. Existen diferentes circunstancias por las cuales evaluamos, en la parte de la enseñanza clínica del pregrado, una parte fundamental es determinar el avance del alumno que le permite, a partir de este proceso, estimular su aprendizaje, ver qué problemas tiene para cumplir los objetivos de aprendizaje y algo fundamental, también para aprobarlo o no. ${ }^{6}$

Después de revisar estos aspectos, ¿̇ómo definimos entonces la evaluación? Como un proceso continuo, sistemático y reflexivo a través del cual se obtiene información cuantitativa y cualitativa pertinente, válida y fiable sobre un objeto, lo que permite identificar fortalezas y áreas de oportunidad para emitir juicios de valor y tomar medidas para perfeccionarlo. ${ }^{?}$

Ahora bien, los principios fundamentales de la evaluación parten de tres puntos:

1. Se debe especificar de manera clara qué se va a evaluar.

2. El procedimiento de evaluación se debe seleccionar para lo que se va a evaluar.
3. Debe tenerse una variedad de procedimientos y usarlos de manera adecuada.

También conceptualizar lo anterior como un medio para conocer un proceso de aprendizaje, mas no un fin en sí mismo. ${ }^{8}$

En la medicina la evaluación debe enfocarse en el perfil de competencias que permita conocer el desempeño del alumno de manera integral en su aprendizaje.

\section{LA EVALUACIÓN TRADICIONAL EN LAS ESCUELAS DE MEDICINA Y ENFERMERÍA EN UN CONTEXTO HOSPITALARIO}

En el contexto de esta pandemia, se llevan a cabo en el instituto múltiples labores docentes tanto de pregrado como de posgrado. En la parte de pregrado se tiene el internado médico por parte de la Facultad de Medicina de la UNAM y la Escuela de Enfermería del instituto incorporada a la Escuela Nacional de Enfermería y Obstetricia de la UNAM. Estos grupos tienen rotaciones clínicas y procesos teóricos que son necesarios de evaluar y que se dificultan al no poder tener contacto directo entre alumno-profesor y alumnopaciente-profesor. De tal manera que es necesario contar con herramientas que permitan conocer el desempeño de los alumnos.

La evaluación teórica de manera cotidiana se llevaba a través de exámenes escritos de opción múltiple, de preguntas abiertas o en algunos casos hasta exámenes orales. Para la parte de la evaluación de la práctica clínica se tenían rúbricas o listas de cotejo para ver las destrezas y conocimientos del alumno al interactuar con los pacientes. Así es como se emitían los juicios de aprendizaje y se realizaban evaluaciones tanto formativas como sumativas.

Pero la vida no se detuvo, las escuelas de medicina y enfermería no pudieron parar los programas. Por tanto, es necesario tener propuestas para evaluar a los alumnos a distancia y tener con ello resultados que nos permitan por un lado, conocer su desempeño académico y por otro, poder realizar las modificaciones y ajustes necesarios a los programas y métodos de impartición de las clases.

En la actualidad la evaluación de los estudiantes de ciencias de la salud en la parte de pregrado se tiene que ajustar a la cuarta generación haciéndose un proceso holístico, que no sólo sea cuantitativo, sino cualitativo haciendo una evaluación completa de las competencias que abarcan lo cognoscitivo, las habilidades mentales, procedimentales y actitudinales con valores y virtudes. ${ }^{9}$

El modelo pedagógico tradicional se basa en las tres primeras generaciones de la evaluación con una postura epistemológica positivista, una relación de profesor-alumno vertical, el proceso de enseñanza basado en la transmisión de contenidos, y por ende, el proceso de aprendizaje es 
meramente memorístico, y para esto la evaluación era en esencia cuantitativa del rendimiento académico. En la actualidad, antes de la pandemia, el modelo pedagógico era básicamente constructivista por competencias, con una postura epistemológica constructivista, una relación profesor-alumno horizontal, el proceso de aprendizaje basado en el aprendizaje significativo con desarrollo de competencias profesionales, el proceso de aprendizaje se establece por construcción de significados y la evaluación básicamente es holística e integral por competencias profesionales con proceso cuantitativos y cualitativos. ${ }^{10}$

A continuación propondremos algunas herramientas de evaluación que podrían ser útiles en estos tiempos de distanciamiento social para evaluar tanto la parte del conocimiento conceptual declarativo como el conocimiento procedimental para grupos de pregrado de medicina y enfermería.

\section{PANORAMA DE RECURSOS TECNOLÓGICOS UTILIZADOS EN LA EVALUACIÓN}

Las Tecnologías de la Información y de las Comunicaciones (TIC) se han constituido en valiosas herramientas para el desarrollo, ya que ofrecen nuevas formas de participación a través de foros y portales de información.

La telefonía móvil promueve el sentido de inclusión, pertenencia y cercanía entre los miembros de una familia o alumnos en las escuelas. Internet es una valiosa herramienta de apoyo a la educación y la investigación al facilitar, por ejemplo, la consulta remota de los catálogos bibliográficos de casi cualquier biblioteca. La computadora permite la creación de documentos electrónicos con una versatilidad inusitada, o el procesamiento de datos en volúmenes anteriormente inmanejables.

La Encuesta Nacional sobre Disponibilidad y Uso de Tecnologías de la Información en los Hogares (ENDUTIH) 2019 tiene como finalidad obtener información sobre la disponibilidad y el uso de las tecnologías de la información y comunicaciones en los hogares y su utilización por los individuos de seis años o más en México. La conectividad a internet en 2019 alcanzó 70.1\% de la población, que equivale a 80.6 millones de personas mayores de seis años (INEGI).

A continuación damos un panorama de la conectividad en el país.

De los 80.6 millones de usuarios equivalentes a $70.1 \%$ de la población, $48.4 \%$ son hombres y $51.6 \%$ mujeres, el grupo de edades que concentra la mayor proporción de usuarios es de 18 a 24 años, 20.1 millones de hogares disponen de internet, esto representa $56.4 \%$ del total de los hogares del país.

El nivel de conectividad del año pasado revela que en los últimos cinco años se han conectado 18,000,000 de personas más, esto equivale a 303,000 nuevas conexiones mensuales. ${ }^{11}$
Lo anterior hace muy complejo que todos los alumnos estén en las mismas condiciones de tecnología de la comunicación, por ello es necesario usar herramientas de mejor acceso.

\section{ALTERNATIVAS DE EVALUACIÓN DEL CONOCIMIENTO TEÓRICO EN LAS ESCUELAS DE MEDICINA Y ENFERMERÍA EN UN CONTEXTO HOSPITALARIO}

En México se adecuaron las plataformas virtuales para dar acceso al grupo de estudiantes y continuar con las clases a distancia. Estas plataformas que pueden ser Zoom, CiscoWebex, WhatsApp, aulas virtuales de Google, Facebook permiten la interacción de los profesores y alumnos, de manera tanto sincrónica como asincrónica, para facilitar los procesos educativos. Esto evidentemente está limitado a los recursos de comunicación con los que cuente el estudiante, y el profesor tendrá que adecuarlos al grupo con el que trabaje. De tal manera que puede ser que la interacción se dé a través de teléfonos móviles o de computadoras.

Desde el punto de vista de la evaluación, en la actualidad el enfoque socioformativo es el que servirá para enfrentar los retos de la pandemia. Este tipo de evaluación diagnostica y facilita el proceso de conocimiento del alumno y también logra la mejora continua en la formación integral del alumno. Se pueden tener ejes de evaluación como los resultados de aprendizaje, problemas y producto, instrumento, valoración, mejora y socialización. Dentro del proceso de evaluación en alumnos de nivel de pregrado de medicina y enfermería es importante valorar los cuatro constructos básicos que son el saber ser, saber hacer, saber conocer y saber convivir, lo que da un aprendizaje integral. ${ }^{12}$

Si bien es cierto que existen diferentes plataformas para evaluar al alumno, no siempre son las ideales para satisfacer las necesidades del mismo. No todos pueden acceder a las tecnologías ni mucho menos adquirirlas, considerando que existen varias que son de paga. Por tal motivo, se propone una herramienta al alcance de todos con un fácil acceso y sobre todo gratuita.

Consideramos que en estos tiempos de pandemia y dadas las condiciones socioeconómicas del país, una de las mejores posibilidades para evaluar la parte del conocimiento declarativo o teórico, el saber conocer y saber ser del estudiante, puede ser el correo electrónico, herramienta muy utilizada en cualquier dispositivo, lugar y hora donde se encuentre $y$, sobre todo, la facilidad de recibir y mandar cualquier tipo de archivo, donde es posible trabajar con el alumno casos clínicos que desarrollen sus habilidades de conocimiento y análisis para la resolución de problemas, y mediante rúbricas el profesor puede evaluar los resultados de los análisis de estos casos.

A través del correo electrónico es posible enviar formularios de Google con exámenes de opción múltiple 
o de preguntas abiertas de forma tanto sincrónica como asincrónica, con lo cual también se pueden evaluar los conocimientos del alumno y toma de decisiones. Con la posibilidad de reenviar al alumno la retroalimentación de sus exámenes y poder tener mejores procesos de evaluación. Estos formularios son ideales de manera sincrónica para poder hacer evaluaciones sumativas.

También es posible elaborar exámenes en Word que el alumno podrá contestar y reenviar al profesor para su revisión. Aquí también surge la inquietud de que el alumno copie o utilice sus textos para contestar el examen, pero aquí son útiles para realizar exámenes formativos y que complementen lo revisado durante las clases.

\section{ALTERNATIVAS DE EVALUACIÓN DEL CONOCIMIENTO PRÁCTICO EN LAS ESCUELAS DE MEDICINA Y ENFERMERÍA EN UN CONTEXTO HOSPITALARIO}

La parte de la evaluación del conocimiento procedimental que determina el constructo del saber hacer al inicio cuando no se tiene contacto con el alumno en cierto escenario como un laboratorio, un quirófano o directamente con un paciente, puede parecer muy complejo, pero hay alternativas fáciles para que el alumno desde su casa apoyado con pocos recursos tecnológicos puede hacer y que nos son de utilidad para su evaluación, considerando también sus limitaciones. Una propuesta es la realización de procedimientos como el enguantado, lavado de manos, realización de suturas en bastidores, algunas exploraciones básicas como toma de presión arterial, oximetría de pulso y también con la posibilidad de realizar algunos experimentos simples, éstos se pueden grabar con un teléfono celular y enviarse para su evaluación a través de plataformas como WhatsApp, o bien, correo electrónico con el apoyo de la nube cuando el video es muy pesado. Se deben proporcionar al alumno las especificaciones y el tiempo del video, para ello se puede mandar una pequeña rúbrica para que el alumno cumpla con ello y nosotros poder emitir una calificación.

También a través de las plataformas de Zoom o CiscoWebex, o bien, videollamadas de Gmail es posible hacer este tipo de evaluaciones de procedimientos simples de manera sincrónica.

El teléfono celular puede ser una herramienta fácil de utilizar y muy intuitiva para el alumno, quien se sentirá con gran confianza para realizar sus videos, con la ventaja de que no es necesario que sea de manera sincrónica para no demorar mucho en caso de grupos grandes.

No estamos descubriendo el hilo negro de esta herramienta, sino más bien tratando de entender al alumno en esta nueva etapa del conocimiento para que tenga acceso a la facilidad, sencillez, simplicidad y comodidad que nos brindan estas herramientas.

\section{CONCLUSIONES}

En un ambiente clínico hospitalario con las limitantes de esta pandemia, la elección de la herramienta tecnológica como apoyo a la docencia dependerá en muchos de los casos de los recursos con los que cuente el alumno y el profesor. Estos recursos deberán ser económicos y de fácil acceso. Y por último, la parte fundamental de la evaluación es saber qué vamos a evaluar para que los resultados que se obtengan nos permitan un adecuado análisis del alumno, del grupo y del curso.

En el pregrado de enfermería y medicina se deberán tomar en cuenta los recursos del alumnado y del propio hospital para adecuar las evaluaciones a estos recursos.

$\mathrm{Y}$ un punto muy importante «evaluamos para enseñar mejor».

\section{REFERENCIAS}

1. Flores HF, Contreras MN, Martínez GA. Evaluación del aprendizaje en la educación médica. Rev Fac Med UNAM. 2012;55(3):42-48.

2. Tyler R. Principios básicos del currículo. Buenos Aires: Troquel; 1977.

3. Escudero T. Desde los tests hasta la evaluación educativa actual: un siglo, el XX, de intenso desarrollo de la evaluación de la educación. RELIEVE. 2003;10(1):11-43.

4. Stufflebeam D, Shinkfield A. Evaluación sistemática: guía teórica y práctica. Barcelona: Paidos; 1987.

5. Graue WE, Sánchez MM, Durante MI, Rivero SO. Educación en las residencias médicas. México: ETM; 2010.

6. Miller GE. The assessment of clinical skills/competence/performance. Acad Med. 1990;65:S63-S67.

7. Martínez GA, Lifshitz GA, Ponce RR. Evaluación del desempeño docente en cursos de especialización médica. Validación de un cuestionario. Rev Inst Mex Seguro Soc. 2008;46(4):375-382.

8. Montiel DI, Lozano SJ, Martínez GA. Evaluación de competencias en ciencias de la salud. México: Panamericana; 2011.

9. Pinilla AE. Las competencias en la educación superior. En: Madiedo N, Pinilla AE. Reflexiones en educación universitaria II. Evaluación. Bogotá: El Malpensante; 2002. pp. 101-135.

10. Guba EG, Lincoln YS. Fourth generation evaluation. Newbury Park, USA: Sage: 1989.

11. INEGI. Encuesta nacional sobre disponibilidad y uso de tecnologías de la información en los hogares. México: 2019. Disponible en: https:// www.inegi.org.mx/temas/ticshogares/

12. Tobon S. La evaluación socioformativa en la educación en línea. En: Luna NJ. Memorias del quinto congreso de evaluación socioformativa (VALORA-2020). México: 2020. Disponible en: https://cife.edu.mx/ recursos

Conflicto de intereses: Los autores declaran no tener conflicto de intereses. 